\title{
La formation professionnelle à l'enseignement entre deux vecteurs intégrateurs en conflit : les disciplines et le curriculum
}

\section{Teacher Training Caught Up Between Two Integrating Vectors: Disciplines and Curriculum \\ La formación magisterial entre dos vectores integradores en conflicto : las asignaturas y el curriculum}

\section{Maurice Sachot}

Volume 28, numéro 2, automne 2000

Réforme curriculaire et statut des disciplines : quels impacts sur la formation professionnelle à l'enseignement?

URI : https://id.erudit.org/iderudit/1080444ar

DOI : https://doi.org/10.7202/1080444ar

Aller au sommaire du numéro

Éditeur(s)

Association canadienne d'éducation de langue française

ISSN

0849-1089 (imprimé)

1916-8659 (numérique)

Découvrir la revue

Citer cet article

Sachot, M. (2000). La formation professionnelle à l'enseignement entre deux vecteurs intégrateurs en conflit : les disciplines et le curriculum. Éducation et francophonie, 28(2), 18-48. https://doi.org/10.7202/1080444ar

\section{Résumé de l'article}

Revenant sur la démarche intellectuelle et institutionnelle qui a présidé à la création des IUFM, en France, en 1989, l'auteur l'identifie comme la mise en oeuvre d'un paradigme historique, celui du curriculum, lequel vient heurter de front un autre paradigme également historique qui préside, depuis

l'avènement de la République, à la formation académique aussi bien scolaire qu'universitaire, le paradigme disciplinaire. Pour rendre compte de ces deux modèles et de l'enjeu de leur affrontement, l'auteur effectue d'abord un long retour sur l'histoire. Leur existence et leur opposition résultent d'une tension première inhérente à l'espèce humaine, à la fois individuelle et sociale. Leur mise en évidence explicite s'est faite en Occident avec l'avènement de la philosophie grecque. La soumission de la logique disciplinaire à la logique curriculaire a été effectuée par le christianisme lorsque celui-ci s'est conçu et construit comme "religion » dans l'espace latin, où la logique curriculaire était prédominante. Les deux paradigmes actuellement en présence sont issus de ce modèle archétypal chrétien, l'un (le curriculaire) par voie de sécularisation (aussi est-il dominant dans le monde anglo-saxon), l'autre (le disciplinaire) par voie de laïcisation (aussi est-il dominant dans le monde catholique et, plus précisément, français). Ce sont deux visions du rapport de la personne à la société qui s'affrontent sur un champ déterminant pour l'avenir : l'école.
Tous droits réservés (C) Association canadienne d'éducation de langue française, 2000 cest protége par la loi sur le droit d'auteur. L'utilisation des services d’Érudit (y compris la reproduction) est assujettie à sa politique d'utilisation que vous pouvez consulter en ligne. 


\section{La formation professionnelle à l'enseignement entre deux vecteurs intégrateurs en conflit : les disciplines et le curriculum}

\section{Maurice SACHOT}

Centre interuniversitaire de recherches interdisciplinaires en didactique (CIRID), Université Marc Bloch, France

\section{RÉSUMÉ}

Revenant sur la démarche intellectuelle et institutionnelle qui a présidé à la création des IUFM, en France, en 1989, l'auteur l'identifie comme la mise en œuvre d'un paradigme historique, celui du curriculum, lequel vient heurter de front un autre paradigme également historique qui préside, depuis l'avènement de la République, à la formation académique aussi bien scolaire qu'universitaire, le paradigme disciplinaire. Pour rendre compte de ces deux modèles et de l'enjeu de leur affrontement, l'auteur effectue d'abord un long retour sur l'histoire. Leur existence et leur opposition résultent d'une tension première inhérente à l'espèce humaine, à la fois individuelle et sociale. Leur mise en évidence explicite s'est faite en Occident avec l'avènement de la philosophie grecque. La soumission de la logique disciplinaire à la logique curriculaire a été effectuée par le christianisme lorsque celui-ci s'est conçu et construit comme « religion » dans l'espace latin, où la logique curriculaire était prédominante. Les deux paradigmes actuellement en présence sont issus de ce modèle archétypal chrétien, l'un (le curriculaire) par voie de sécularisation 
(aussi est-il dominant dans le monde anglo-saxon), l'autre (le disciplinaire) par voie de laïcisation (aussi est-il dominant dans le monde catholique et, plus précisément, français). Ce sont deux visions du rapport de la personne à la société qui s'affrontent sur un champ déterminant pour l'avenir : l'école.

\section{ABSTRACT}

\section{Teacher Training Caught Up Between Two Integrating Vectors: Disciplines and Curriculum}

Maurice SACHOT

University Marc Bloch, France

The author finds, in the intellectual and institutional approach that led to the creation of the IUFMs (teacher colleges) in France in 1989, the implementation of a historical paradigm, that of the curriculum. This paradigm came to collide head on with another historical paradigm, that of the disciplines, which has reigned over high school and university training since the earliest days of the Republic. The author undertakes a full survey of these paradigms to take full account of their meaning and the issues involved in their conflict. Their existence and their opposition are seen to result from a tension inherent in humans, both as individuals and as social creatures. This conflict first came clearly to light in the Western world with the beginnings of Greek philosophy. The rationale for curriculum took then precedence over the rationale for the disciplines through the efforts of the Christian church, which was created and established as the religion of the Latin world, in which curriculum dominated. The two paradigms as they exist now come from this Christian archetype. The paradigm for curriculum comes by way of secularism, which held sway in the AngloGermanic world. The paradigm for the disciplines comes by way of laicization, which helds way in the Catholic world, or more precisely, France. These two visions of how individuals relate to society are doing battle in an area that will prove decisive for the future, the schools. 


\section{RESUMEN}

\section{La formación magisterial entre dos vectores integradores en conflicto: las asignaturas y el curriculum}

Maurice SACHOT

Universitdad Marc Bloch, France

Revisando el enfoque intelectual e institucional que presidió la creación del IUFM en Francia en 1989, el autor lo identifica como la operacionalización de un paradigma histórico, el del curriculum, el cual se confronta con otra paradigma asimismo histórico que preside, desde la instauración de la República, la formación académica tanto escolar como universitaria, es decir, el paradigma de las asignaturas. Para repertoriar los dos modelos y los retos que surgen de dicha confrotación, el autor realiza un largo periplo histórico. Su existencia y sus oposiciones surgen de una tensión inherente de la especie humana, tanto individual como social. En Occidente, dichos modelos se explicitan con el advenimiento de la filosofia griega. La subordinación de la lógica de las asignaturas a la lógica curricular fue llevada a cabo por el cristianismo al momento de ser concebido y construido como una «religión » en el espacio latino, espacio en el donde la lógica curricular era predominante. Dichos paradigmas surgen del modelo arquetípico cristiano : uno (el curricular) surge por la via de la secularización (por ello es dominante en el mundo anglosajón), el otro (el de las asignaturas o materias ), por la via de la laicización (dominante en el mundo católico y especificamente, francés). Se trata de dos visiones de la relación entre la persona y la sociedad que se confrontan en un espacio clave para futuro : la escuela.

\section{Introduction}

Le propos d'ensemble de cette contribution est de porter un regard critique sur la façon ou tout au moins sur l'une des façons les plus répandues dont nous essayons aujourd'hui de penser et d'organiser la formation professionnelle à l'enseignement. Si l'on voulait en effet décrire celle-ci, telle qu'elle s'effectue actuellement au travers des diverses institutions qui en ont la charge, on serait certainement bien en peine d'en faire une présentation cohérente et, plus encore, de dire si elle relève d'un modèle précis et duquel il s'agit. On parviendrait sans doute à identifier des sousensembles assez bien constitués, des logiques suffisamment claires ici ou là à l'œuvre. Mais l'image dominante qui en résulterait serait plutôt celle d'un patchwork, d'un empilement disparate, d'un tout incohérent sinon contradictoire.

Aussi bien ne perd-on pas de temps à entreprendre cette analyse. On décrète d'emblée qu'il est impossible de penser et de modéliser la formation à partir de ce 
qu'elle est et qu'il est vain de vouloir s'appuyer sur cette modélisation pour soumettre les systèmes de formation à l'examen et envisager s'il convient de procéder par refonte ou par réforme. La solution qui s'impose d'elle-même - et qui commande en fait l'attitude précédente - est de repartir d'une tout autre base, totalement neuve, de construire $a b$ initio un curriculum de formation à partir de ce qui est supposé la finaliser tout en la transcendant, à partir du projet qu'elle est censée servir: l'enseignement effectué auprès des élèves et des étudiants. Par une analyse descriptive du milieu de l'enseignement, il est possible, croit-on, de dégager un référentiel professionnel complet et cohérent, puis, par déduction logique, de modéliser le curriculum de formation qui doit être en amont. Tel est le parti qui a été pris pour refonder en France la formation des maîtres et qui a donné lieu, à la fin des années 1980, à la création des IUFM (Instituts universitaires de formation des maîtres). Le paradigme qui préside à leur constitution suit, de manière explicite, une logique curriculaire intégrale avec, pour outil, un référentiel professionnel et de formation (voir le rapport du recteur Bancel 1989).

Or, ce paradigme, même s'il n'est pas entièrement nouveau, s'oppose de manière radicale et brutale au modèle qui, en France, depuis une bonne centaine d'années, préside explicitement non pas à la formation des maîtres de l'enseignement primaire, mais à la formation universitaire des enseignants du secondaire : le paradigme disciplinaire. Mais la France ne fait pas figure d'isolée. Par ailleurs, ce qui est advenu dans la formation à l'enseignement et qui atteint désormais l'ensemble des formations universitaires elles-mêmes (voir Sachot, 2000a) est l'aboutissement d'une tendance générale qui a pris naissance dans la formation professionnelle avant de s'emparer de la formation initiale.

Un tel engouement mérite cependant réflexion. Le renversement que la logique curriculaire propose dans la façon de problématiser la formation soulève de sérieuses critiques. Il n'est pas sûr, en particulier, qu'il sorte du modèle idéologique que, justement, il récuse et entend dépasser, ni ne surmonte, par le recours à des catégories intégratives (comme celle de métier, de profession, de compétences, d'interdisciplinarité, d'action...), des oppositions que ce modèle implique, telles que les oppositions entre théories et pratiques, savoirs savants et savoirs d'action, savoirs disciplinaires et savoirs professionnels. Peut-être même est-il de nature à les radicaliser ou, plutôt, à les occulter comme problèmes tout en s'en servant comme fondements. Aussi bien est-ce ce nouveau paradigme que nous nous proposons d'interroger en le confrontant au paradigme disciplinaire qu'il supplante et réduit à sa merci $^{1}$.

1. Cette étude prolonge d'autres que nous avons menées sur la catégorie de discipline scolaire, dont ces deux qui abordent le rapport entre discipline et référentiel: Sachot, 1998a et 1998c (à paraître). 


\section{Les termes du conflit}

\section{Les graves insuffisances d'une formation}

À l'origine de ce conflit, on peut facilement y voir le fait que, jusqu'à l'avènement des IUFM, la formation professionnelle à l'enseignement n'avait pas, dans la formation à l'enseignement secondaire et à l'enseignement supérieur, pris en compte l'enseignement lui-même auquel elle prépare, les conditions dans lesquelles celui-ci s'exerce, les personnes auxquelles il s'adresse, les objectifs et les finalités qu'il poursuit, les institutions et les milieux dans lesquels il s'insère. Et cela, malgré les nombreux travaux, de tous ordres et de toutes disciplines, qui ont été effectués depuis au moins un siècle (depuis la sociologie jusqu' aux didactiques, en passant par la psychologie et toutes les sciences pertinentes en éducation). La fonction enseignante était encore trop généralement perçue et vécue comme une conséquence naturelle, donc non formalisable et non explicitement formalisée, de la qualité intrinsèque de l'enseignant, comme le rayonnement solaire est l'effet naturel de son incandescence interne. Omne bonum diffusivum sui, « tout bien diffuse de lui-même». Si donc la lumière n'atteint pas tel ou tel objet, ce n'est pas la source qui est en cause. Celle-ci est à chercher soit du côté de l'objet, qui se met hors de sa portée, soit du côté du milieu intermédiaire qui s'interpose au lieu de transmettre. Le maître est maître de sa discipline, non de ses élèves, de leurs capacités et de leurs dispositions, ni du milieu institutionnel, politique et scolaire, ni du milieu social et familial de ses élèves ou étudiants.

\section{Les deux modèles en présence}

D'entrée de jeu, le conflit et sa résolution se sont donc présentés comme l'affrontement, largement implicite, entre deux modèles fondamentaux. Le premier, que nous qualifions de disciplinaire, envisage le cursus de formation (curriculum) sur la maîtrise d'une ou de plusieurs disciplines. Celles-ci sont le moteur principal, le vecteur intégrateur et explicite qui, au moins en principe, donne force et cohésion à l'ensemble de la formation, le rendant autosuffisant. L'autre modèle, à l'inverse, envisage le même curriculum de formation à partir de toutes les tâches que l'enseignant est amené à effectuer et donc de toutes les compétences et capacités, si disparates soient-elles, qu'il doit maîtriser pour les assurer. Aucune logique de cohérence interne, si ce n'est celle de la pertinence et de l'efficacité. Dans cette perspective, les disciplines font donc plutôt figure d'obstacles. Si elles peuvent encore constituer des sous-ensembles indispensables pour donner une cohérence intellectuelle à des savoirs déterminés, elles sont en revanche un obstacle majeur dans la mesure où elles sont accusées de morceler cette formation et, plus grave encore, dans la mesure où elles prétendent à l'autosuffisance, en se définissant par elles-mêmes et pour elles-mêmes et non pas à partir du projet de formation qu'elles sont supposées servir. Toutes les critiques que l'on formule contre les disciplines peuvent d'ailleurs être ramenées en définitive à cette raison première.

Ce clivage profond entre disciplinarisme et curriculumisme n'est pas totalement nouveau, tant s'en faut. On pourrait aisément montrer que, dans l'espace 
français tout au moins, il est à l'œuvre dans l'opposition que l'on a établie depuis la Révolution française entre les deux modèles fondamentaux auxquels on recourt pour penser globalement la formation scolaire: celui de l'instruction et celui de l'éducation (voir Sachot, 1997; Sachot 1998c; Lelièvre, 1999). Le premier est porté par la logique disciplinaire, même si le mot n'y est pas, et le second par le milieu qui finalise les savoirs, disciplinaires ou non. Le premier définit historiquement le curriculum du lycée républicain, celui de l'enseignement général, ainsi que la préparation des enseignants, qu'habilite à enseigner la réussite à un concours (agrégation ou CAPES), lequel est totalement - ou presque totalement depuis peu - un savoir disciplinaire. Le second modèle, celui de l'éducation, définit traditionnellement le primaire et la formation des maîtres qui enseignent à ce niveau (les anciennes Écoles normales), le lycée napoléonien et toutes les formations à caractère professionnel (primaire supérieur autrefois, lycées et filières techniques et professionnels aujourd'hui, etc.).

\section{Vers une exacerbation du conflit}

Mais l'évolution récente atteste plutôt un raidissement dans les positions que d'un assouplissement. L'alternance (entre études et stages), comme mode de gestion pédagogique des formations professionnelles, y compris celle des enseignants, signe l'incompatibilité entre les deux modèles. Plus encore, la victoire que semblent près d'emporter les partisans de la référentialisation sur ceux des disciplines est plus de nature à exacerber le conflit qu'à lui mettre un terme. Ces derniers, qui ont l'appui des universitaires dont les disciplines sont enseignées au primaire et au secondaire, ne sont sans doute pas prêts à passer sous les fourches caudines, à accepter ce qu'ils considèrent comme étant une dévaluation de leurs disciplines, dévaluation perçue en outre comme un asservissement. L'atteste, même si c'est intenable épistémologiquement, la mainmise qu'ils gardent jalousement sur la didactique de leurs disciplines. Peut aussi être convoquée comme témoin la création des IUFM que nous avons déjà évoqués: c'est une institution extra-universitaire et non intérieure à l'université. Le caractère universitaire qui les qualifie signifie sans doute la prise en compte de l'incapacité, sinon du refus de l'Université d'intégrer dans la formation à l'enseignement qui lui était traditionnellement dévolue la part qu'exige la prise en considération des conditions dans lesquelles celui-ci s'exerce; mais il signifie tout autant la volonté d'intégrer la recherche universitaire, de l'instrumentaliser et de la mettre au service de ses propres desseins. Même si, depuis leur fondation, les IUFM se sont vu attribuer quelques-unes des prérogatives proprement universitaires disposer de postes d'enseignants-chercheurs (c'est-à-dire d'universitaires) qui ne soient pas affectés à l'Université voisine et pouvoir procéder eux-mêmes au recrutement en ayant leurs propres commissions de spécialistes -, la possibilité de conduire des programmes de recherche proprement dite et de constituer à cette fin des équipes reconnues et financées par l'État n'est pas encore acquise, tant s'en faut. Si elle l'était, ce ne serait qu' «en éducation ", c'est-à-dire non pas avec le statut de recherche fondamentale, mais seulement comme recherche appliquée, comme cela se fait dans le domaine des sciences exactes. Remarquons enfin que, contrairement 
à l'Université, l'instance décisionnelle des IUFM, son conseil d'administration, est présidée par le recteur d'académie en personne, modalité institutionnelle qui signifie clairement que tout ce qui se fait dans l'IUFM est instrumentalisé au service de l'institution. Ainsi donc, quelle que soit, dans le concret, la qualité des relations entre les deux types d'institutions et entre les personnes, le clivage tend plutôt à s'accentuer qu'à se réduire.

\section{Une nécessaire réflexion}

Il s'avère donc nécessaire de prendre toute la mesure des deux modèles en présence. Comme ils ne sont pas des modèles a priori, ayant chacun un étalon déposé au ciel des idées, mais qu'ils sont le résultat actuel d'une longue histoire particulière, il est nécessaire, pour ce faire, de déconstruire cette construction historique pour mettre si possible en évidence la logique ou les logiques qui ont présidé à son élaboration.

L'histoire des mots, toujours déterminants, sera notre meilleur guide. L'usage des deux termes, «disciplines » et "curriculum», que nous mettons en exergue pour appréhender l'enseignement et la formation qui y prépare, est très récent. L'emploi du premier, celui de "disciplines ", parce qu'il est la reprise du latin disciplina et qu'il appartient désormais à la langue courante, peut sembler multiséculaire, voire bimillénaire. En fait, il ne date que d'une bonne centaine d'années. Il n'est pas d'origine scientifique et est entré sans que l'on s'en aperçoive, comme c'est souvent le cas pour les mots qui ont le plus d'importance ${ }^{2}$. Mais il s'est généralisé à une telle vitesse, déclassant ou rendant archaïques les autres termes, qu'il en est devenu banal, au point qu'il semble presque avoir perdu toute consistance, sauf lorsqu'il s' agit de trouver un bouc émissaire pour porter tous les péchés du système éducatif. Le médiologue dira cependant que c'est dans cette banalité même que réside justement sa force principale.

L'usage du second dans l'espace français est encore plus récent, au point même de sonner étrangement aux oreilles d'un pur francophone: malgré sa forme et sa consonance latines, il ne figure pas en effet avec ce sens ou un sens plus ou moins équivalent chez les meilleurs auteurs latins. La tradition latine a, dans cet emploi, plutôt légué des termes comme educatio, institutio, cultus, cultura et, en toute première place - c'est à noter -, disciplina. Cet usage de « curriculum » vient en réalité de l'espace linguistique et culturel anglo-saxon, où "discipline» n'a guère de pertinence. L'usage ordinaire de «curriculum» pour appréhender l'éducation serait de peu postérieur à celui de "disciplines» dans l'espace français. Le terme aurait été adopté par «les pionniers anglo-saxons de l'Éducation nouvelle, à commencer par Dewey» (De Landsheere, 1992 : p. 89). L’ouvrage qui est considéré comme fondateur d'une science du curriculum est de celui de Bobbitt, The Curriculum (1918). Mais, dit V. De Landsheere, «on considère généralement que la première formulation moderne

2. Ce qu'après A. Chervel (1988), nous avons mis en évidence et précisé dans plusieurs de nos études (Sachot, 1993, 1996, 1997, 1998c). 
de la théorie du curriculum se trouve dans les Basic Principles of Curriculum and Instruction de Tyler (1950) ». Ainsi donc, si le terme de " disciplines » est bien français et sa charge sémantique non directement liée à une investigation scientifique, celui de «curriculum» est en revanche bien anglo-saxon et spécifie une catégorisation plutôt scientifique.

Cette première observation, d'ordre linguistique, est majeure. En effet, les deux termes ne renvoient pas d'abord à deux concepts théoriques qui pourraient transcender les espaces culturels, tels des concepts mathématiques. Ce ne sont pas que deux outils conceptuels qui, n'ayant de pertinence et de force qu'au sein de la théorie qui leur donne existence, seraient neutres et donc opérationnels, comme les outils techniques, dans n'importe quel espace culturel ${ }^{3}$. Ils sont chacun gros d'une histoire politique, culturelle, institutionnelle et intellectuelle propre, l'anglo-saxonne et la française. Peut-être même en sont-ils les emblèmes quand il s'agit de la formation. La venue du terme «curriculum » dans l'espace français ne saurait donc être réduite au seul rôle d'un outil conceptuel, d'un concept que l'on pourrait manipuler et intégrer sans difficulté majeure. Il se peut que telle soit, à terme, la conséquence de la confrontation avec le modèle français, si celui-ci résiste positivement, c'est-à-dire se montre suffisamment puissant pour se transformer ${ }^{4}$. Mais rien ne permet de préjuger du résultat. Seule l'histoire le dira.

\section{Retour sur la longue histoire}

À la vérité, l'opposition qu'enregistrent les deux termes «disciplines » et «curriculum " dans l'approche de la formation, opposition à laquelle l'enracinement dans deux modèles culturels emblématiques donne sa pleine mesure, n'est que la forme actuelle d'une tension constitutive de l'espèce humaine en même temps que l'héritière de formes concrètes qu'a prises cette tension au cours de l'histoire en Occident.

\section{L'éducation et la formation, vecteurs de deux logiques de sens opposés}

Les origines nous échappent, tout comme les centaines de milliers d'années qui séparent les origines d'une époque où l'humanité est historiquement saisissable ${ }^{5}$. Peut-être même conviendrait-il de remonter encore plus en amont, si l'analyse des comportements animaux les plus proches de notre espèce n'est pas trop entachée d'anthropomorphisme. Quoi qu'il en soit, l'homo sapiens sapiens est, par nature, un

3. Sur cette distinction à faire entre technique et culture, voir les fines analyses de R. Debray (1997).

4. On chante volontiers les charmes et les beautés des "métissages culturels ». Que les cultures soient ouvertes les unes aux autres et, plus encore, que les hommes y trouvent leur identité est une nécessité. À condition que ce ne soit pas un embrouillamini indigeste. Ce n'est pas parce que le collier à cheval et l'arbre à cames répondent tous deux à la définition d'organe de transmission de l'énergie qu'ils sont interchangeables! On sait le coût que représente l'échec récent d'une sonde envoyée vers Mars en raison d'un mélange entre les $\mathrm{km}$ et les miles. Les distinctions qui valent pour la technique valent aussi, mutatis mutandis, pour la culture.

5. Des travaux comme ceux d'A. Leroi-Gourhan $(1943,1945,1970,1974)$ ouvrent cependant des fenêtres lumineuses sur l'aube de notre civilisation. 
homo educatus educans. L'éducation et la formation sont à la fois une qualité et une contrainte de l'espèce humaine. La raison en est que l'homme est, indissociablement, un être individuel et collectif. Un individu ne peut, seul, naître, grandir, se développer et vivre. De la même manière, l'humanité, en tant qu'espèce, ne peut s'organiser et se maintenir sans les individus qui la composent. Or, c'est à la jonction de ces deux exigences premières, pour banales qu'elles puissent paraître, que se forme, nécessairement, un troisième terme: l'éducation et la formation. Il est ce médiat, fût-il informel, qui les vectorise l'une par rapport à l'autre, qui catalyse leurs oppositions en même temps que leur complémentarité et qui tend à s'envisager sous la forme de deux curriculums fondamentaux de sens opposés, selon que l'on part plutôt de l'homme individuel ou plutôt de l'homme collectif.

Cette vectorisation est, sous ses deux formes, la meilleure et la pire des choses. Fondée de part et d'autre sur la dynamique du profit et du don, de la contrainte et de la liberté, de la violence et du respect, elle donne la capacité à chacune des deux logiques de se retourner contre elle-même et contre l'autre partie avec une force égale au bienfait qu'elle peut et doit apporter. Chacune peut chercher à ne viser que ses propres fins, et, pour cela, instrumentaliser l'autre, comme elle peut chercher à servir librement les fins de l'autre. L'effet pervers n'est pas une conséquence fâcheuse accidentelle, comme l'effet secondaire d'un médicament. Il n'est pas d'abord dû aux circonstances extérieures dans lesquelles la relation s'exerce. Il réside dans la relation elle-même, dans le principe même de toute vectorisation. Ne sont de mise ni l'angélisme (tout le monde est bon) ni le manichéisme (même sous la forme atténuée que l'on prête à Rousseau, gauchissant sa pensée: "L'homme naît bon, c'est la société qui le déprave»).

\section{Épistèmè et paideia}

\section{L'avènement de l'épistèmè, " discipline "}

Telle est la vectorisation originaire qui fonde, depuis toujours, ce qui fut un jour explicitement identifié par les deux catégories qu'aujourd'hui nous désignons et appréhendons par les termes de discipline et de curriculum. Dans l'histoire occidentale, si ce n'est dans l'histoire universelle, cet événement est datable, l'identification de l'un induisant nécessairement celle de l'autre. Cela s'est passé dans l'espace grec avec l'apparition de la philosophie en tant que telle, c'est-à-dire comme discipline de recherche, comme posture épistémologique, prise de distance, position en surplomb (épistèmè: se tenir au-dessus) par rapport au réel, pour d'abord le connaître et énoncer un jugement de valeur (cognitif, esthétique ou éthique) à son propos (ce qui implique, par discrimination interne, une multiplicité quasi indéfinie d'épistèmai, de «disciplines»). Dans la pensée d'un Platon et de ses successeurs philosophes, la discipline, l'épistèmè, devient le précieux fil d'Ariane pour remonter au principe, à l'archè, à ce point de départ originel d'où il est ensuite possible de redescendre vers le monde, dont l'éducation - la paideia ${ }^{6}$ - fait partie, pour le saisir dans son ensemble,

6. En grec, paideia reste nébuleux : sa signification fondamentale est " qui concerne un enfant » (le statut de l'enfance, son régime de vie et d'éducation...), un pais (enfant d'homme libre comme enfant d'esclave), quelqu'un qui n'a pas la majorité ou qui, comme l'esclave, n'a pas le statut d'homme libre, statut qui définit la véritable majorité. 
en définir les finalités et en organiser le parcours, bref, en faire la théorie. Cette activité de théorisation est avant tout une activité de connaissance. Sa logique est une logique de scientificité, même si, bien évidemment, ses critères et sa méthodologie ne sont pas exactement ceux des nos actuelles sciences expérimentales (Hippocrate fait cependant état d'expériences au sens moderne du terme, non seulement du point de vue méthodologique, mais encore par le fait qu'il exclut a priori le hasard la tychè - comme principe d'explication). Même si la connaissance peut être une fin en soi, elle vise aussi à former le jugement de l'homme libre, qui veut connaître pour devenir meilleur (et donc heureux) et pour rendre meilleure la cité à laquelle il appartient. Elle se déploie donc principalement sous la forme d'un curriculum personnel, comme élaboration et choix d'un style de vie, d'un idéal à atteindre, en conformité avec ce que l'on croit être l'essence de l'homme (voir Hadot, 1995).

\section{Son vecteur principal : la langue}

Le vecteur premier auquel est due l'émergence de la catégorie explicite d'épistèmè est la langue grecque elle-même, dont l'une des potentialités est de faire surgir et d'identifier comme tels, en les substantivant, les notions et les concepts. Cette potentialité est d'abord offerte par l'usage de l'article défini et de l'article défini devant une forme verbale, avant tout de l'infinitif. Elle est encore portée par une distribution du système verbal qui lui fait saisir le procès plus sous son aspect que selon son inscription temporelle. Or, la catégorie de l' «aoriste " (de l'« indéterminé ») permet de saisir l'idée verbale "pure ", comme concept. Enfin - mais cet inventaire n'est pas exhaustif -, la langue grecque ne distingue pas nettement entre le singulier et le pluriel dans le genre neutre, dans ce genre qui, justement, permet d'appréhender les choses selon leurs catégories ${ }^{7}$ : to eipein, «le dire» (aoriste, distinct du présent to légein, «le fait de parler»), par exemple, permet de saisir la parole, ho logos, en tant que concept et non pas seulement comme action ou chose. To dikaion, «le juste», c'est désigner la catégorie du juste, l'idée du juste, l'essence du juste, lesquelles sont distinctes de la justice comme pratique judiciaire (hè dikè) ou comme sentiment ou vertu (hè dikaiosynè). La langue grecque oriente donc elle-même vers une appréhension du monde qui distingue entre l'essence des choses, fondatrice, générale et universelle, et le réel, perçu alors comme accidentel, divers, variable et labile (voir Benveniste, 1966). Cette essence est, à l'inverse du réel, insaisissable par les sens. Elle est comme le centre du cercle ou de la sphère qui se définit à partir de la circonférence (comme en latin, dont est issu notre terme « définir », le mot qui, en grec, signifie « définition» a, pour sens premier, « limite», ho horos). Et la détermination du centre s'obtient par une démarche hypothético-déductive, sans briser le cercle ou la sphère. Mais, une fois celle-ci effectuée, il devient logiquement possible de construire la figure mathématique du cercle ou de la sphère à partir du centre ${ }^{8}$.

7. Quand le sujet est un neutre pluriel, le verbe reste au singulier. Une forme d'adjectif à l'accusatif neutre équivaut à un adverbe sans qu'on puisse faire une distinction entre le singulier et le pluriel (kakon et kaka équivalent à kakôs «mal»).

8. Aussi bien, pour un grec, la mathématique devient-elle la forme par excellence de l'activité par laquelle la connaissance (gnôsis) se construit, s'acquiert et s'enseigne (mathèsis, mathèma). 
Ce qui n'est pas exactement un retour vers le réel, encore moins d'un retour sous la forme de l'actualisation d'une potentialité ou de l'«application» d'une théorie.

À l'intérieur du vecteur de la langue, la prise de parole et sa mise en scène sur l'agora, liées à l'organisation en cités sédentaires, ainsi que le développement de la rhétorique pour éclairer le jugement et la prise de décision, voire en rendre raison, ont puissamment contribué au questionnement proprement philosophique, à s'interroger sur la nature du juste ou du pire au nom desquels on justifie telle ou telle décision, personnelle ou collective.

\section{La tentative de Platon}

Il importe de remarquer que cette démarche intellectuelle, s'effectuant selon une logique de scientificité, est un questionnement qui, même s'il repose sur une démarche personnelle, porte, de manière indissociable, sur la personne et sur la société, sur la nature du rapport entre l'individu et sa cité, entre ses compétences et ses qualités personnelles et la bonne administration de celle-ci. Tous les dialogues de Platon pourraient être ici convoqués. Mais deux d'entre eux mettent plus particulièrement cette question en relief. Le premier est Alcibiade ${ }^{9}$. Le rapport y est abordé plutôt à partir de l'individu, de son épistèmè, de la maîtrise (comme ensemble de compétences et comme maîtrise de soi) que doit avoir quiconque entend prendre la parole dans l'assemblée démocratique pour éclairer ses décisions. Qu'est-ce qui habilite le jeune Alcibiade à se lancer dans une carrière politique? À l'issue d'un premier examen de ses compétences, il résulte que «l'énumération des différentes compétences techniques nécessaires à la bonne administration de la cité permet d'établir que le meilleur conseiller, en une matière donnée, est celui qui possède le savoir adéquat; en ce sens et selon les circonstances, c'est le technicien spécialisé qui devrait seul recevoir la parole à l'assemblée» (Pradeau, 1999 : p. 35-36). Ce que n'est pas Alcibiade, comme ne le sont d'ailleurs pas les autres membres de l'assemblée, tous hommes libres, donc non définis par l'exercice et la maîtrise d'une technè. D'où un déplacement de la question, qui conduit à l'affirmation que « seule une connaissance peut fonder la compétence recherchée» (Ibid., p. 41), puis que cette connaissance et la maîtrise de soi sont l'œuvre, si l'on suit le précepte delphique («connais-toi toimême»), non de la cité et de ses membres, mais du sujet lui-même.

C'est à toi-même qu'il revient de prendre soin de toi-même, en commençant d'abord par te connaître toi-même. Ce ne sont pas des maîtres de vertu ou des dirigeants politiques qui pourront prendre soin des jeunes gens, ce ne sont ni des rhéteurs, ni des parents, ni des oracles, ni même des pédagogues compétents qui pourront leur enseigner qui ils sont. Si une maîtrise de soi est possible, si un engagement politique peut l'être à son tour, le jeune homme doit en être le sujet (Ibid., p. 52-53).

9. Lire ce dialogue dans la nouvelle traduction faite par Chantal Marboeuf et Jean-François Pradeau, traduction qu'enchassent une excellente introduction et des notes fort précieuses faites par ce dernier (Marboeuf et Pradeau, 1999). 
À cette affirmation de la priorité du sujet individuel, à la fois comme fin de l'épistèmè (connaissance et maîtrise de soi) et comme base de la construction de la cité, répond celle de la priorité de la cité, au bien et à l'amélioration de laquelle doivent concourir tous les individus, lesquels en seront en retour les véritables bénéficiaires. C'est La République ${ }^{10}$, dialogue dans lequel le philosophe, grâce à l'épistèmè, construit l'idéal type de la cité juste et détermine explicitement, comme à partir de ce centre, un curriculum de formation pour tous ceux qui en composent les parties. Une observation importante doit être faite. L'activité à laquelle se livre Platon n'est plus une activité d'épistèmè, de recherche, mais de programmation, d'opérationnalisation. Si la première est scientifique, la seconde ne l'est pas. C'est le politikos qui pense et agit et non plus le théôrètikos, le théoricien. Plus précisément, en investissant dans cette entreprise curriculaire les outils et les résultats de sa propre recherche, il les met au service d'autres vecteurs qui sont à l'œuvre, vecteurs qui sont en grande partie implicites et que le discours savant, en se mettant lui-même en avant, rend plus implicites encore. En effet, le curriculum qu'il élabore comme $a$ priori et fait reposer sur un principe remarquable, celui de la justice, peut être considéré, pour une grande part, comme une rationalisation du curriculum réel des principaux groupes ou fonctions sur lesquels repose la cité grecque d'alors : la paideia n'est pas la même, en particulier, selon que l'on est un homme libre (un sujet) ou que l'on est un esclave, pour qui la formation se réduit à l'acquisition et à la maîtrise de compétences (technai), excluant la maîtrise des finalités de ses compétences (lesquelles appartiennent à son maître et propriétaire, l'homme libre).

\section{Un avertissement}

Peut-être est-ce là le piège le plus grave que cache le rapport entre l'épistèmè comme science ou discipline et le curriculum comme plan de formation. Prétendre construire $a b$ ovo un curriculum de formation à partir de ce que la science peut en dire, c'est sombrer dans le totalitarisme, parce que c'est donner à un projet, nécessairement factuel et limité, la puissance d'un vecteur que libère totalement la suppression de sa propre finalité (connaître) pour en faire un outil de rationalisation. L'épistèmè peut, comme corpus disciplinaire (outils et résultats), être mise au service d'un vecteur d'un autre ordre ou relevant d'un autre niveau de discursivité (comme l'ordre politique). Mais elle perd alors sa qualité d'épistèmè. Sa finalité n'est plus la connaissance. Dans ce nouvel emploi, elle n'a aucune fonction fondatrice ${ }^{11}$. La décision qu'elle instruit a en elle-même son proprement fondement. Présenter cette

10. La traduction française, qui reproduit la traduction latine, gauchit le titre grec, Hè politeia, qui signifie « qui concerne la polis» (la cité), le régime sociopolitique (quel qu'il soit) qui détermine la vie des ses membres en tant que citoyens (politeis).

11. Toutes les raisons qu'on pourrait avancer pour fonder quoi que ce soit sont en général moins sûres que ce qu'elles sont censées fonder, a fait observer Wittgenstein (1976, article 307, p. 83). 
décision sous le masque de l'épistèmè, de la science, et se servir de celle-ci comme vecteur d'énonciation, de justification, de validité ou de légitimité est une imposture ${ }^{12}$.

\section{De l'épistèmè grecque à la disciplina chrétienne}

Dire que le rapport entre discipline et curriculum est déjà entièrement joué avec Platon serait grossir les origines de ce qui est advenu par la suite. Le curriculum de La République n'est jamais entré dans les faits ${ }^{13}$. Il est resté un exercice intellectuel. Manquait pour cela un lien institutionnel intrinsèque avec la cité. Ce lien fut noué par le mouvement chrétien, lorsque celui-ci s'est compris comme religio.

\section{Res Romanae ou la spécificité de l'épistémologie latine}

La romanité, en effet, est le premier milieu par lequel la culture grecque nous a été transmise, même pour ceux qui estiment aujourd'hui en être les héritiers directs, comme le peuple grec ${ }^{14}$. Dire cela, c'est d'abord affirmer, contrairement à l'opinion reçue, qu'il existe une philosophie latine et, donc, en ce qui concerne le rapport que nous étudions, une façon spécifiquement latine de l'envisager, qu'ensuite ce milieu impose sa marque à la pensée grecque qu'il transmet, qu'enfin - pour nous en tenir à ce qui nous paraît majeur - le christianisme a noué d'une façon spécifique les deux conceptions latine et grecque.

\section{La langue latine comme vecteur}

La langue latine a la même origine indo-européenne que la langue grecque. Elle en diffère cependant de beaucoup. Si nous reprenons, par exemple, les mêmes potentialités de la langue que précédemment, nous constatons qu'elle n'a pas d'article, que son système verbal distribue le procès presque uniquement sur une linéarité temporelle et que le nombre est pertinent au neutre. Elle n'est donc pas portée, comme l'est la langue grecque, à orienter la saisie des choses par la médiation conceptuelle et explicite de leur essence. Le Latin est donc concret. Si le Grec cherche l'archè, l'origine ou, peut-être plus exactement, l'originaire qui, à la fois, transcende le monde sensitif et l'histoire, le Latin se situe d'emblée dans le monde des choses (res) et de leur cours. Il ne les quitte jamais. S'il y a une transcendance, c'est dans l'immanence du monde qu'elle réside, dans la capacité reconnue au monde et aux hommes de se construire eux-mêmes. C'est à l'homme de déterminer, de dire (fatum, que fausse la traduction par «destin» en l'assimilant à tychè) de

12. L'autorité de jugement ou de décision et l'autorité de savoir relèvent de deux ordres qui ne peuvent être confondus : la qualité de la décision d'un chef de gouvernement ne résulte pas de sa compétence dans une discipline donnée, si importante soit-elle en politique, comme il est mensonger de justifier la décision d'une assemblée ou d'un gouvernement par une argumentation scientifique.

13. Même si Platon, homme d'action, a cru pouvoir passer à l'acte en se rendant en Sicile pour faire l'éducation de Denys le Jeune. L'idée du prince-philosophe sera un thème récurrent.

14. Ce n'est pas parce que, aujourd'hui, nous pouvons encore lire dans le texte original les textes grecs qu'il est fondé de concevoir notre rapport à la culture grecque antique selon une tradition linéaire et continue. Ce serait rester dans une vision chrétienne, même sécularisée, de l'histoire, construite sur le concept d'accomplissement (voir M. Sachot, 2000b). 
manière arbitraire, dans une linéarité indéterminée, le moment précis où il fait commencer sa propre histoire, son initium (qui n'est pas davantage une archè) $^{15}$. Ce point de départ est la fondation de Rome ( $a b$ Urbe condita). Si, pour le Grec, l'idée ou l'essence est ce centre invisible qui fonde le cercle, pour le Latin, ce centre est une réalité bien concrète: Rome. «Rome est un centre qui définit une périphérie» (Eco, 1986: p. 31). L'histoire romaine (res romanae: «les choses romaines ») n'est qu'une séquence temporelle. Si elle a un début, elle aura une fin (les Latins étaient millénaristes) ${ }^{16}$.

\section{L'homme maître de son destin}

Cette vision, que l'on peut qualifier de pessimiste, puisqu'elle n'est ni traversée ni portée par un projet qui la transcende, est contrebalancée par le pouvoir d'action reconnue aux choses du monde elles-mêmes, et aux hommes en tout premier lieu, pouvoir dont on ne cache pas la violence. La mythologie que propose Virgile des origines latines pour les inscrire dans une visée transcendante est purement littéraire. Mais elle reste réaliste. S'il y eut jamais un âge d'or, puis un âge d'argent, l'âge romain - l'histoire réelle - commence avec l'âge d'airain (aeneus), c'est-à-dire par l'âge du métal qui fait violence aux autres (les armes, arma) et aux choses (le socle de l'araire qui creuse le sillon qui délimite l'espace (voir le récit fondateur - fratricide - de Rome) ou féconde le champ (la sédentarisation) ${ }^{17}$. L'homme idéal (le vir), dans cette perspective, est alors un héros, construisant sa propre destinée comme celle de sa cité. L’histoire est un récit de gesta, de facta, bien situés dans le temps (elle prend la forme d'annales), non un débat d'idées. Le poète a parfaitement compris sa propre culture, lorsqu'il nomme son héros fondateur de Rome Énée ("d'airain») et fait commencer sa fiction par ces deux mots énoncés de manière indissociable : Arma uirumque...

Cet état de violence ne repose pas sur une vision manichéenne du monde. Pour le Latin, le monde est bon. Le mal, la malignité, la haine n'ont pas plus de réalité ontologique que le reste. Ils sont dans l'histoire, de l'histoire: odi «je hais» est, comme noui «je sais », un thème de parfait, non de présent. Ils supposent une antériorité temporelle, un événement qui s'est produit dans l'histoire, non un fondement inscrit dans la nature. Pour le Latin, l'homme est donc maître de son destin, de « dire » (fatum) ce qu'il veut en faire, à la différence du Grec qui se voit plus volontiers emporté par une tychè transcendante. L'histoire d'une même racine indoeuropéenne, *smer, est, à cet égard, révélatrice: le thème qui, en indo-européen, signifie "part», se connote, en grec, de l'idée de «échue par le destin» (la mö̈ra est un autre nom de la tychè et devient synonyme d'anagkè, la "nécessité »), donc ce sur quoi l'individu n'a aucune prise et, en latin, de l'idée inverse de "acquise par l'individu " (meritum, mérite, notion qu'il faut chercher en grec du côté de l'arètè, de l'excellence, traduit ordinairement dans un autre registre par virtus).

15. Pour une présentation plus ample et plus précise de ce que nous développons dans ce paragraphe, nous nous permettons de renvoyer à Sachot (1989).

16. Non au sens gnostique du terme, mais au sens où ils acceptaient d'emblée que leur propre histoire aurait une fin après un laps de temps relativement long, comme elle avait un début.

17. D'un point de vue historique, l'invention de l'airain est bien antérieure à la fondation de Rome. Mais en associant la naissance de Rome à cet âge (mythique), les Romains, réalistes, reconnaissaient, comme le fait aujourd'hui le médiologue, les dépendances mutuelles entre techniques (artes) et culture (cultura). 


\section{Une épistémologie fonctionnelle}

D'où l'épistémologie fonctionnelle qui caractérise la connaissance du Latin: c'est en modifiant le monde (dont il fait partie) que l'homme connaît et se connaît, et non en l'observant, comme le fait le Grec ${ }^{18}$. D'où également l'importance reconnue à l'institué et aux institutions, à ce qui détermine le droit et l'éthique dans cette transformation $^{19}$. Un exemple pris dans le monde contemporain fera comprendre cette profonde différence : si notre physique relève d'une épistémologie grecque (au moins jusqu'à une période très récente), notre chimie relève d'une épistémologie latine. Mais dépendants d'une épistémologie grecque, nous sommes incapables de faire l'épistémologie de la part latine de notre culture, part de plus en plus importante, et notre droit et notre éthique courent toujours derrière, sans jamais rattraper ni accompagner ni, encore moins, précéder les transformations du monde auxquelles nous procédons. Notre concept de nature (natura est latin) qui commande réellement notre pratique est en fait saisi sur le plan conceptuel dans celui de physis (qui est grec).

Nous ne pouvons mieux faire, nous semble-t-il, pour bien mettre en évidence les clivages qui séparent la pensée latine de la pensée grecque, que de reprendre les huit oppositions que Dumézil établissait entre les Romains et les Indiens (voir Dumézil, 1966 : p. 123-124). Ce qu'il disait de ces derniers peut, en effet, être dit des Grecs :

- Les Romains pensent historiquement alors que les Indiens pensent fabuleusement [...].

- Les Romains pensent nationalement et les Indiens cosmiquement [...].

- Les Romains pensent pratiquement et les Indiens philosophiquement [...].

- Les Romains pensent relativement, empiriquement ; les Indiens pensent absolument, dogmatiquement [...].

- Les Romains pensent politiquement, les Indiens pensent moralement [...].

- Enfin, les Romains pensent juridiquement, les Indiens pensent mystiquement $[\ldots]$.

La rencontre entre la culture grecque et la culture romaine peut logiquement conduire à toutes sortes de figures: juxtaposition, intégration de l'une par l'autre, mixage plus ou moins incohérent, etc. Dans le domaine éducatif, la présentation que fait H.-I. Marrou laisse plutôt penser que «s'il y avait à Rome une tradition pédagogique originale [...], l'éducation latine a évolué dans un tout autre sens, car Rome

18. Dans l'épistémologie grecque, le chercheur s'abstient de modifier le monde (dont les personnes font évidemment partie) pour le connaître. Il le respecte. De manière absolue. Quitte à déployer des trésors d'imagination pour connaître son objet sans y toucher. C'est sur ce qu'on dit du monde et sur la représentation qu'on en a qu'il travaille, non sur le réel lui-même (episkepsômetha ti legomen, " examinons de près de ce que nous disons » : telle est la méthode que Socrate formalise [Platon, Euthryphron, 7a]).

19. Persona (d'où « personne » en français) n'a pas le sens du grec prosôpon, dont pourtant il provient. II identifie I'individu par son appartenance à un groupe, par les droits et les devoirs que cette appartenance lui confère. Rappelons que, pour un Grec, la personne humaine reste un indéterminé, un point d'interrogation (tís). Elle ne saurait être comprise à partir des définitions de tous ordres qu'elle reçoit dans la société, définitions qui sont des formes de réduction en même temps que de reconnaissance. Elle reste ce visage visible, mais insondable, que je scrute du regard (prosôpon), masque (même mot: prosôpon) qui cache tout autant qu'il révèle. 
s'est trouvée amenée à adopter les formes et les méthodes de l'éducation hellénistique» (Marrou, 1965 : p. 356).

Nous dirions plus volontiers que, si elle a adopté, pour reprendre les termes d'H.-I. Marrou, les formes et les méthodes de l'éducation hellénistique, Rome n'en pas pour autant abandonné sa perspective fondamentale, perspective qui, à l'inverse de la grecque, fait dépendre la cité (ciuitas) du citoyen (ciuis), de son engagement et de sa responsabilité personnels, de sa fides et de sa pietas $^{20}$. L'ouvrage de Platon, Hè Politeia, est traduit par res publica, la «chose publique». Ce qui n'est pas pareil. Paideia, en revanche, ne se laisse pas saisir par un seul équivalent en latin, car la réalité concrète apparaît trop multiforme pour être ainsi catégorisée. L'activité de connaissance, à la différence de l'épistèmè grecque, est plutôt saisie dans sa pluralité concrète. Elle est à la fois une activité sur soi et sur le monde, une activité que m'imposent les exigences de la vie personnelle et collective. Elle est plurielle, indistinctement disciplinae et artes.

Ce rapport très étroit qui lie concrètement le citoyen à sa cité, les philosophes latins l'explicitent en investissant un terme capable de l'exprimer dans toute son ampleur, terme sans équivalent dans les autres langues: religio. Et c'est de ce mot que va dépendre, comme d'un sésame magique, par suite de l'entrée en lice du christianisme, une refonte des relations entre culture grecque et culture romaine.

\section{La disciplina chrétienne}

Le mouvement chrétien comporte intrinsèquement une dimension «religieuse ». Mais c'est une erreur de considérer que, dès le départ, il était une "religion ». Il l'est devenu. Et seulement au terme d'un long parcours et en donnant à ce mot sa propre définition ${ }^{21}$. Ce parcours fut effectué en trois étapes majeures.

\section{Accomplissement du judaïsme}

Le mouvement chrétien, comme chacun sait, s'est constitué au sein du judaïsme, c'est-à-dire, pour ce qui nous concerne ici, au sein d'un peuple qui place son identité sous l'autorité d'un Dieu unique, d'un être transcendant qui intervient dans le temps du monde et des hommes pour y accomplir son dessein, y établir son règne, dans son peuple élu et, par son peuple, dans le monde. Pour accomplir ce curriculum collectif et historique qui fait de lui un instrument de son Dieu, le peuple d'Israël se doit d'inviter chacun de ses membres à se conformer à la Loi qu'il dit être donnée par Dieu lui-même. Ce curriculum collectif est premier, mais suppose l'adhésion de chacun. S'il donne lieu à des interprétations multiples et variées, l'interprétation la plus répandue dans le peuple juif au début de l'Empire romain est

20. Pas plus que, plus d'un millénaire plus tard, par suite d'une nouvelle entrée de la philosophie grecque (le Nouvel Aristote) dans l'0ccident latin par la médiation des Arabes, les théologiens ne feront une théologie grecque ou orthodoxe. Aristote est instrumentalisé au service d'une théologie latine. Il permet d'affirmer, contre Saint Augustin (le Latin converti) et en faveur de Pélage (resté bien latin), que la grâce est éduite des potentialités de la nature (voir Sachot, 1991).

21. Ce que nous énoncons ici très schématiquement a fait l'objet d'un livre auquel nous nous permettons de renvoyer le lecteur (Sachot, 1998b). 
celle que promeut le mouvement pharisien, notamment grâce aux assemblées synagogales où il a fait introduire la lecture des Prophètes après celle de la Loi (au sens strict du terme). Cette lecture des Prophètes accentue une relecture historique (attente de l'avènement du Règne de Dieu), spirituelle et intériorisée (personnelle) de la Loi.

Cette proclamation scripturaire synagogale est, selon nous, le berceau du mouvement chrétien, la structure sociotechnique qui lui permet de prendre corps. En proclamant que le temps présent est le moment de l'accomplissement des Écritures (la venue du règne de Dieu), le temps de l'homélie, Jésus et ses disciples après lui font du mouvement que l'on qualifiera de chrétien avant tout un discours de vérité, non pas au sens philosophique du terme, mais au sens d'accomplissement authentique de l'attente du peuple juif (même si ce n'en était qu'une partie), telle qu'une certaine écoute des Prophètes pouvait en permettre l'élaboration conceptuelle. Le propos initial du mouvement chrétien n'est donc pas de fonder une nouvelle société, mais d'appeler chacun des membres du peuple juif à aller jusqu'au bout de son appartenance au peuple élu en adhérant personnellement à l'interprétation qu'il donne du temps présent et en en assumant les implications comportementales.

L'échec dans sa tentative à convaincre l'ensemble du peuple et, surtout, ceux qui président à ses institutions conduit le mouvement chrétien à s'identifier essentiellement comme curriculum de vie personnelle, curriculum qui repose sur un respect de la Loi juive (au moins au début) et sur une adhésion volontaire de chacun (signifiée par le baptême). Une nouvelle sorte de société prend donc ainsi forme, société non pas fondée sur la naissance, mais entièrement sur l'adhésion libre de ses membres, lesquels ne peuvent être dès lors que des adultes. Non nascuntur christiani, fiunt, «On ne naît pas chrétien, on le devient », écrira plus tard Tertullien.

\section{Philosophie révélée}

La seconde étape est consécutive à la diction du donné et du discours chrétien en langue grecque et, plus précisément, lorsque le mouvement chrétien fut pensé par ceux dont la langue était uniquement la langue grecque et dont la culture de formation était grecque (soit exclusivement, soit juive et grecque à la fois). Il est alors appréhendé comme une école de philosophie. Cette nouvelle saisie ne modifie pas ses bases constitutives, à savoir de reposer sur un discours de vérité et une adhésion individuelle. Mais elle les renforce singulièrement en lui donnant la forme et la force de la pensée philosophique, dont il fait sa servante.

Le changement le plus important vient du retournement que cette saisie fait subir à ce nouveau vecteur en même temps qu'au type de discours qui est le sien. Pour un philosophe grec, la vérité, parce que construction purement humaine, est irrémédiablement marquée au coin du doute et de l'incertitude. Fruit d'une quête inachevée et tâtonnante de l'homme, elle reste une doxa, une "opinion", même élaborée comme épistèmè. Dans le discours chrétien, l'épistèmè change de nature: elle est transformée en vérité dogmatique et absolue. En affirmant que le discours qu'ils tiennent ne vient pas des hommes, mais de Dieu lui-même, les chrétiens le 
transforment en Vérité révélée, donc, non critiquable, non discutable, s'imposant par elle-même, aussi bien au niveau personnel que collectif.

\section{« Religio »}

À cette étape de son développement, le mouvement chrétien, même organisé en communautés, n'a pas une inscription sociale et politique suffisante pour que la société tout entière soit informée, au sens aristotélicien du terme, par cette épistèmè chrétienne. Cette possibilité devint effective lorsque, troisième étape importante dans la genèse du christianisme occidental, il s'énonça en latin et s'inscrivit, grâce à la qualification de religio que proposa, par un coup de force proprement inimaginable, Tertullien, en 197, comme forme symbolique de l'institué romain. Il put alors se construire avec tout l'institué social et politique que représentait le monde romain. Il n'était plus seulement une épistèmè révélée, définissant essentiellement un curriculum personnel fait d'adhésion conceptuelle (la foi) et de rectitude comportementale (conformité à la Loi). Il prit aussi la puissance d'un curriculum politique, au sens complet du terme.

Très exactement, il établit la collusion entre trois instances jusqu'alors distinctes :

1. La personne, autonome et responsable de ses projets et décisions (curriculum personnel).

2. Le système intellectuel par lequel chacun s'efforce de construire du sens et du vrai (curriculum intellectuel ou proprement disciplinaire).

3. Le système politique, enfin, qui définit l'intérêt commun et, selon les différents niveaux d'organisation de la société qui le composent, l'ensemble des tâches, fonctions et missions réclamant des compétences multiples et spécifiques des individus (curriculums professionnels, institutionnels, etc.).

Religio devient, dans la bouche des chrétiens, le terme générique qui désigne à la fois l'institué ecclésial comme disciplina totale et véritable, c'est-à-dire à la fois comme règle (regula), comme doctrine (doctrina, qui reprend épistèmè dans sa nouvelle valeur spécifique, mais qui se soumet à toutes les disciplinae, comme des servantes, ancillae) et comme démarche personnelle (fides, foi, engagement personnel, façon de vivre). L'articulation entre ces trois vecteurs constitutifs n'est pas aléatoire. L'épistémologie de «révélation" dans laquelle la religio christiana «religion chrétienne » s'énonce entend justifier la relation hiérarchique qui est imposée à tous : l'institution commande à la doctrine et, par elle, détermine l'engagement et le comportement de chaque croyant, lequel devient le véritable monde qu'il s'agit de transformer, puisque le salut (plus personnel que collectif) dépend de cette transformation $^{22}$.

La forte intégration faite par la religion chrétienne entre ces trois vecteurs n'interdit pas la possibilité de leur distinction: en s'affirmant comme doctrina (comme science totale), même révélée, l'institution ne peut raturer complètement

22. Ce qui est une reprise et un infléchissement de l'épistémologie latine. L'Histoire appartient à Dieu. Mais le salut dépend de l'histoire de chacun. 
l'interrogation philosophique qu'elle a retournée et dont elle a besoin pour se formuler. De même, elle ne tait pas, mais au contraire continue de faire proclamer dans les célébrations liturgiques le message évangélique qui suscite normalement l'adhésion du croyant et qui est directement une contestation du pouvoir institutionnel (y compris clérical). Mais il faudra attendre un millénaire pour que cette distinction commence à se faire jour et produise ses effets, engageant la pensée et les institutions occidentales dans un long processus qui, limité à la partie latine de la chrétienté et non encore achevé, va prendre deux formes, l'une de sécularisation, l'autre de laïcisation, chacune de ces deux formes définissant un espace culturel propre où prennent justement naissance les deux conceptions qui s'opposent dans notre façon d'envisager l'éducation et la formation: celle à laquelle se rattache le terme de curriculum, et celle à laquelle se rapporte celui de disciplines.

\section{Curriculum ou disciplines}

La sécularisation et la laïcisation de la société christianisée participent d'un même processus qui, commun à tout l'Occident latin (mais encore étranger à l'Orient chrétien, c'est-à-dire à la partie grecque - et à ses dépendances - issue de l'Empire Romain chrétien, en bref, aux pays orthodoxes), peut être globalement défini comme un relâchement dans la liaison entre les trois vecteurs constitutifs de la religion chrétienne ou, positivement, comme l'autonomisation (relative) des personnes, de la pensée et des institutions, quelles qu'elles soient, par rapport à une autorité religieuse, réduite, sur le plan institutionnel, à une association intérieure à la société (à une Église) et, sur le plan intellectuel, à la valeur d'une opinion (voire une superstition). Sa dynamique n'est pas extérieure à la religion chrétienne, mais naît de la tension entre ses trois vecteurs internes. Si sa genèse est longue et se manifeste sous différentes formes (autonomisation du pouvoir politique - voir la querelle des Investitures entre 1075 et 1200; autonomisation de groupements sociaux ou de classes sociales - voir l'avènement de la bourgeoisie, des communes et des corporations; autonomisation de la pensée - voir l'avènement de l'Université, etc.), sa problématique générale peut être analysée comme un retournement de la structuration antérieure: c'est une prise de position épistémologique (vecteur intellectuel) qui affirme qu'il revient à l'individu, à la personne (vecteur personnel) de déterminer sa propre conception des choses (vecteur intellectuel) et d'organiser la société comme elle l'entend (vecteur institutionnel), et non plus à celle-ci de déterminer la doctrine et le comportement de chacun.

L'épistémologie qui est donc supposée fonder cette position est une épistémologie grecque retrouvée, mais limitée à ce qui peut être "scientifiquement " prouvé, la croyance et la conviction échappant, comme objets d'investigation, à la logique de scientificité. C'est une épistémologie élaborée dans les facultés de théologie des premières universités, au XIII ${ }^{\mathrm{e}}$ siècle, et qui permet au doctor (non à tout un chacun) de formuler une science théologique (théoriquement) indépendante en tant que telle du discours de la foi (et donc de l'autorité proprement ecclésiastique), parce 
qu'énoncée dans un discours d'un autre ordre, le discours savant (voir Sachot, 1991). Et nous ne sommes toujours pas véritablement sortis de cette limitation première qui préserve la croyance et les institutions religieuses, restreignant la démarche épistémologique à sa conception «scientifique» et s'entretenant sous la forme d'un débat convenu entre la science et la foi, la société civile et la religion, etc.

\section{De la sécularisation à la prédominance du curriculum La sécularisation protestante}

Sur cette base, la conception d'ensemble des rapports entre personne et société va prendre deux directions majeures. La première, de sécularisation proprement dite, est celle qu'enregistre et accomplit tout à la fois l'avènement du protestantisme, lequel, pour être un mouvement de toute la société, n'en reste pas moins une contestation à l'intérieur de l'Église chrétienne latine. Trois traits principaux le caractérisent : le premier est l'extension à tout individu de la capacité à élaborer lui-même sa propre doctrine (le libre examen). Celui-ci n'a pas besoin, pour cela, de la médiation d'une institution (l'Église constituée) pour lui dicter la Vérité et l'exacte interprétation des textes et du monde. Dieu seul (la grâce, sola gratia) y suffit. Le second, qui est le corollaire du premier, est la capacité reconnue à chaque individu de s'organiser en sociétés (et donc en églises) comme il l'entend (d'où l'éclosion des « sectes » protestantes et la conception multicommunautariste ou multiculturaliste de la société). Le troisième, enfin, est un retour sur l'épistémologie antérieure pour confondre les deux ordres de discours que sont ceux de la science et de la foi. La conception protestante ne résulte donc pas simplement d'une inversion de la religion chrétienne, telle qu'elle a été définie plus haut. Son épistémologie, qui est une épistémologie de la croyance et non de la science, emportée par son mouvement contre les abus des institutions ecclésiastiques, finit par faire complètement l'impasse sur le vecteur institutionnel, à réduire le rapport entre le sujet et le monde à deux termes et donc l'intelligence du second par le premier à une adcequatio intellectus et rei, une "adéquation entre l'intelligence et le monde», sans autre instance médiatrice que l'interprétation ellemême que le sujet se donne de la Bible (et, plus largement, du monde ${ }^{23}$.

Parce que, d'une part, il occulte la fonction proprement instituante des institutions sociopolitiques et que, d'autre part, il n'établit pas une distinction nette entre épistémologie de la foi et épistémologie de la science, le protestantisme induit un mouvement de sécularisation dans lequel il n'y a pas de véritable rupture entre la «religion» et la société, un monde où, finalement, la meilleure société est celle qui «accomplit» le message évangélique, celle où la religion s'accomplit en s'abolissant dans la société, comme le levain dans la pâte, rendant impossible la distinction entre «religion» et société. Une théocratie sans Église, en quelque sorte, ou, plutôt, une société qui est en même temps une Église. Cuius regio, eius religio: la religion d'un pays est celle de celui auquel il appartient. La référence à Dieu, représenté par la

23. L'herméneutique est, en toute rigueur de terme, la seule «discipline institutionnelle » possible en milieu protestant. Paul Ricœur en est le plus éminent représentant actuel en milieu français. 
Bible, lue individuellement, mais proclamée collectivement, empêche la société de s'écrouler sous sa propre immanence.

Si Dieu lui-même inspire chaque individu et l'interpelle au plus profond de sa conscience, si chacun fait sien le jugement de Dieu, la société peut alors être, pour reprendre le titre à un livre de Jürgen Habermas (1991), la société des individus. Et chacun est libre de constituer son curriculum personnel, aussi bien de style de vie que professionnel. Mais c'est faire preuve de trop d'optimisme, pour ne pas dire d'angélisme, à moins de recourir cyniquement à la théorie de la prédestination, d'un fatum divin (non humain) qui décide $a$ priori du sort de chacun. C'est considérer que l'homme, comme Adam, naît adulte dans un environnement favorable et qu'il est uniquement animé par des pulsions et des sentiments qui le poussent à ne vouloir que le bien. C'est instaurer comme étant équitables, parce qu'inscrites dans la nature par Dieu, les inégalités entre les individus, et entre les individus et le monde. C'est oublier que chaque individu naît enfant, que l'éducation est une nécessité absolue et qu'elle ne peut être laissée à sa libre initiative.

\section{Une société auto-instituante}

Et qu'advient-il si, le mouvement de sécularisation allant à son terme, le monde lui-même, la société instituée étaient donnés à la place de Dieu et de la Bible? Aboutissement inévitable, étant donné l'absence d'une institution spécifique exprimant explicitement ce fondement transcendant. Le monde lui-même et l'individu sombreraient alors dans un déterminisme ou un immanentisme encore bien plus fermé que celui du monde romain antique, puisque l'on donnerait à ce monde la fatalité du divin transcendant, de ce qui s'impose à chacun comme destin personnel et collectif. En niant la fonction d'institutions médiatrices et instituantes, données explicitement comme telles et sommant sans cesse les individus et les organisations humaines de justifier leurs actions et leurs décisions, cette conception laisse en fait à la société le redoutable pouvoir d'être auto-instituante. La société des adultes ne repose pas, en définitive, sur l'ensemble des individus, mais sur quelques personnes qui détiennent, directement ou indirectement (comme le détenteur de capital, nomade des temps modernes), les organisations de tous ordres qui composent la société, qui ont ou recherchent la maîtrise des choses et des hommes, non forcément leur bien, et qui n'ont pas forcément à justifier devant tous leurs décisions. Invoquer, comme le faisait Adam Smith (1776), une "main invisible» providentielle rééquilibrant constamment les rapports entre les individus et la société revient à donner libre cours à n'importe quel type d'entreprise, sans s'occuper des conséquences fâcheuses qu'elle peut entraîner, puisqu'une régulation est supposée se faire automatiquement.

\section{Le curriculum comme acculturation}

La sécularisation achevée conduit donc, en définitive, à inverser le rapport sur lequel elle se dit fondée. Ce n'est plus à l'individu, mais à la société elle-même, telle qu'elle est, que revient le soin de déterminer le curriculum d'éducation et de formation. Totalement perçu à partir du monde des adultes, aux fonctions qu'exercent les 
adultes dans la société, ce curriculum ne peut être au service de chaque individu. Définie par des tâches à exercer, la formation réduit l'individu à ses compétences et à ses ressources. Réduction que l'individu fait sienne comme définition personnelle par l'éducation qui lui est proposée. En effet, déterminée par le milieu auquel il appartient (milieu social, profession, entreprise, communauté ou association de toutes sortes), l'éducation est avant tout une acculturation, l'inscription forte dans une appartenance qui s'impose à lui comme une donnée de fait. Dans cette perspective, les disciplines ne peuvent être qu'instrumentalisées: savantes en amont ou savoirs constitués pour le formé, elles sont mises au service de son acculturation et de l'acquisition de compétences, depuis leur description jusqu'à la mise en œuvre du curriculum de formation. Cela n'y change rien, mais au contraire l'aggrave, que des savants de tous bords cautionnent cette emprise de la société sur les individus en « démontrant » que ce qu'elle propose (vend), y compris la formation, répond à leurs besoins ou à leur attente. De ce point de vue, la théorie "socioconstructiviste " dont on se réclame actuellement pour penser les formations, professionnelles ou initiales, en matière de curriculum, mériterait d'être soumise à un examen sérieux. Elle fait l'impasse sur la fonction instituante, sur l'instance médiatrice qui détermine les exigences et les compétences attendues (comme si le fait social était un droit et un devoir), sur ceux qui les identifient, sur les procédures utilisées et sur les passages qui conduisent de cette analyse à la proposition d'une formation, de sa validation et de sa certification. La loi du marché n'est pas à elle-même son propre fondement.

\section{De la laïcisation à la prédominance des disciplines} La laïcisation en milieu catholique

À la sécularisation à laquelle le protestantisme imprime sa marque profonde s'oppose en partie, mais sur des points essentiels, le mouvement de laïcisation, lequel s'est développé en milieu catholique, et plus spécifiquement en France (mais non dans les pays méditerranéens), à partir du moment où le protestantisme s'est implanté de façon majoritaire dans la plupart des pays nordiques. La différence essentielle tient au fait que le mouvement de laïcisation est un mouvement politique et non religieux, même si la religion y est fortement impliquée. Se construisant dans un espace catholique, dans un espace où la part des institutions y est prégnante, le mouvement de laïcisation cherche à marquer nettement les seuils, les ruptures en même temps que les relations, entre les trois vecteurs sur lesquels reposait jusqu'alors la formation chrétienne. La laïcisation n'en abolit aucun. Elle n'envisage pas la vie humaine selon une relation bipolaire entre l'individu et la société. Elle considère que l'un et l'autre, pour exister et se construire, ont nécessairement besoin de la médiation d'instances instituantes et définies comme telles. Ce qui la conduit, même si la démarche est loin d'être achevée, à opérer des distinctions, non seulement entre l'individu (curriculum personnel), le niveau épistémologique (curriculum intellectuel) et la société (curriculum professionnel ou autre), mais, encore, au niveau de la société elle-même, entre ses différentes composantes (depuis l'appartenance à des ethnies jusqu'aux associations de tous ordres, en passant par les professions, les religions, etc.), et ses définitions globales, mais non identiques, que sont la nation, la 
patrie, l'État et la République. Si chacune de ses composantes a sa légitimité, la société n'est pas pour autant considérée comme instance instituante : c'est à la République, et à la République seule que revient cette prérogative.

\section{La fonction instituante de la République}

Mais cette République-là n'est pas une Église. Elle n'est détentrice d'aucune vérité. Et c'est même très précisément sur ce point qu'elle s'oppose au christianisme. Son point d'ancrage principal est d'ordre épistémologique. Si elle est l'instance instituante de la société, ce n'est pas en tant qu'institution - elle n'est pas une institution -, mais parce qu'elle n'est rien d'autre que les citoyens eux-mêmes. La République transcende toutes les institutions qui la composent, parce qu'elle est faite des individus-citoyens qui, en tant que personnes libres, douées de raison, capables de jugement personnel et responsables, transcendent tout ce qui les constitue (qualités et déficiences personnelles) comme toutes les appartenances ou inscriptions qui les identifient concrètement (familiales, sociales, professionnelles, religieuses, etc.). À la différence des institutions qui lui donnent corps et où les intérêts et les rapports de force sont souvent premiers, la République est cette instance où les citoyens définissent les principes auxquels ils se soumettent euxmêmes et auxquels est soumis tout ce qui s'élabore dans la société, y compris les lois votées démocratiquement. Sa force tient à la capacité reconnue de ses membres à dépasser le niveau de leurs intérêts particuliers pour chercher ensemble le bien commun. De ce point de vue, elle est et reste en grande partie utopique ${ }^{24}$.

\section{La discipline comme vecteur de la forme scolaire de l'éducation républicaine}

Ce qui ne l'est pas, ce sont les rapports que cela induit dans les relations entre les individus et la société. Dans le projet républicain, le regard sur l'individu et la société n'est pas angélique. L'un et l'autre sont sans doute bons par nature, mais d'une bonté qui est à construire. L'individu y a la priorité, car tout repose sur lui. Il est d'abord un homme, et non une ressource humaine pour la société. L'individu naît enfant et, même devenu adulte et responsable de ses actes, il reste un être fragile. Son éducation est une nécessité à laquelle la société des adultes ne peut se soustraire. Et cette éducation concerne d'abord l'être même de la personne et non seulement ses aptitudes ou compétences sociales. Mais elle ne saurait prendre la forme d'une inculcation idéologique ou d'un quelconque asservissement. Ce qui serait contradictoire. L'inscription de chacun dans un espace qui l'inculque n'est pas niée. Mais l'épistémologie sur laquelle repose la visée républicaine, épistémologie fondamentalement grecque dans son inspiration - avec sa dimension éthique indissociable -, refuse de considérer cette situation de fait comme un destin ou un droit. Au contraire, elle considère que seul l'usage d'une logique de scientificité est légitime dans la transformation à laquelle elle invite l'enfant-être-au-monde pour qu'il devienne un adulteêtre-au-monde. «L'école républicaine ne délivre pas de messages. Elle délivre tout

24. Il est clair, au vu de cette définition, qu'il ne suffit pas d'appeler un État « République » pour qu'il le soit effectivement! c'est un idéal dont même la France ne s'est pas vraiment approchée. Actuellement, elle s'en éloigne même à grands pas. 
court» (Debray, 1992: p. 60). D'où la construction du curriculum de formation initiale à partir des disciplines (l'apparition du mot avec ce sens est liée à l'avènement durable d'une forme républicaine de l'État). La discipline est même l'unique vecteur de la forme scolaire de l'éducation républicaine ${ }^{25}$.

Le résultat ne peut donc être garanti: il n'est pas sûr que l'enfant, soumis à l'instruction, atteigne ce niveau de culture (personnelle et non celle qui l'assimile à un groupe déterminé, comme dans le système précédent) qui fait véritablement de lui un adulte. Agent premier de son instruction en même temps que le premier bénéficiaire, c'est à lui, et à lui seul, que revient (et reviendra) la responsabilité de porter un jugement sur lui et le monde ${ }^{26}$. Il n'empêche. Si donc la formation à l'enseignement vise à former des personnes qui auront pour tâche de former des enfants, elle ne saurait se déduire d'une observation du métier d'enseignant, ni de l'attente ou des besoins de ses élèves ou, derrière eux, de l'attente des parents ou de la société. Destinée avant tout à former des citoyens qui auront à former des citoyens, elle doit être traversée de part en part par la logique de scientificité.

\section{Les limites de la logique disciplinaire}

L'impasse sur l'implicite inculcant

Cette visée républicaine de l'école et de la formation qui y prépare est-elle sans défaut? Non. Focalisée sur l'instrumentation intellectuelle (la raison) qui, seule, peut aider l'enfant à se libérer de lui-même et de l'emprise de la société27, elle fait l'impasse sur l'individu réel et sur le monde réel. Plus exactement, trop confiante dans les individus et dans les résultats de la mise en œuvre de la logique de scientificité, elle n'est pas allée jusqu'au bout de sa démarche. Quand la forme politique

25. Dans cette perspective, la discipline scolaire, selon la définition que nous avons déjà donnée ailleurs (Sachot, 1997), définit le milieu institué (concrètement, une configuration de disciplines), par lequel le milieu instituant (la République), en exerçant des enfants-êtres-au-monde (les élèves), dans un environnement si possible adapté (établissement, classe, activités...), avec l'aide d'adjuvants spécialisés (les maîtres ou professeurs) et selon une logique procédurale spécifique (logique de scientificité qu'atteste la forme énonciative faite par le maître et/ou les élèves) vise à les former comme adultes-êtres-au-monde (personnes capables d'un jugement libre et responsables).

26. Comme on peut le relever d'une analyse des programmes scolaires depuis les débuts de la III République jusqu'à une date récente (la perspective curriculaire tend à y mettre fin), une discipline scolaire - et c'est ce qui la constitue comme telle - poursuit trois niveaux d'objectifs: cognitifs, culturels et éducationnels. En régime de chrétienté et de sécularisation proprement dite, les objectifs éducationnels (porter un jugement quant au vrai, au beau et au bien) sont les premiers et ont un contenu spécifique, défini par l'institution ecclésiastique ou la société. Les objectifs culturels ont aussi un contenu : la conformation à la culture chrétienne ou à la communauté à laquelle on appartient (religieuse, sociale, politique, sportive, éducative, professionnelle, etc.). Les objectifs intellectuels, enfin, sont réduits à la plus simple expression pour le fidèle: un catéchisme (religieux ou non) sur lequel il n'a aucune prise. En régime républicain, tout l'effort porte sur les objectifs intellectuels, sur ce qui instruit la personne et l'arme pour la vie. La culture est presque vide de contenu, car elle se définit de manière personnelle (être un homme cultivé, qui a du recul par rapport au monde dans lequel il vit) et non de manière collective (appartenance à une communauté). Les objectifs éducationnels, enfin, sont sans aucun contenu : il revient à chacun de se faire et de porter un jugement (voir Sachot, 1994, p. 57-61).

27. C'est en ce sens que l'école républicaine est "libérale ", comme on le disait au siècle dernier. La liberté qu'elle promeut ne se confond pas avec le caprice. Elle ne se définit pas, comme des maîtres l'enseignent réellement dans des classes, comme "la liberté de penser, de dire et de faire tout ce que l'on veut ", ni ne s'arrête quand commence celle d'autrui. Cette liberté-là est celle que fait miroiter la société marchande, une société qui renvoie à l'individu une image qui flatte ses désirs, ses fantasmes, voire ses pulsions pour mieux lui vendre les moyens de les assouvir. La liberté véritable est celle qui permet à chacun d'avoir d'abord la maîtrise de lui-même, parce qu'il est suffisamment instruit et capable de porter un jugement réfléchi sur luimême, sur le monde et sur son action dans le monde. 
de l'État est devenue durablement républicaine, en 1880, il n'y a pas eu en effet de remise à plat, comme en 1789. La République s'est inscrite dans des institutions et une société qui n'étaient pas républicaines. Les citoyens et leurs représentants n'ont pas véritablement soumis à la sagacité de la raison leurs multiples niveaux d'organisation, en particulier ceux que sont, à une échelle globale, la nation, l'État et la patrie ${ }^{28}$. Concrètement, la République, c'est aussi tout cela. Et ce « tout cela », parce qu'il est laissé dans l'indéterminé, définit et régule tout autant que les disciplines les curriculums réels de formation, qu'ils soient individuels ou collectifs, initiaux ou continués, implicites ou explicites, informels ou formels, etc., identifiés comme tels ou sous d'autres appellations par les savants ${ }^{29}$. Ce n'est pas le moindre mérite de l'entrée de la notion de curriculum dans le champ éducatif français que de mettre le doigt sur cet implicite par lequel l'élève est « discipliné », c'est-à-dire inculqué. Car si sa catégorisation est nouvelle, ses formes concrètes sont bien anciennes, identifiées depuis longtemps sous des termes comme cursus (des études), ratio studiorum, programmes (d'études), plan (d'études) et, plus simplement, études, et plus spécifiquement encore, primaire, secondaire, supérieur, filière, voire école, collège, lycée, université, grandes écoles, IUT, IUP... La notion explicite de curriculum atteste que, même sans être nommée à ce niveau d'appréhension, la fonction curriculaire est bien présente chez les individus, dans la société et dans l'école et qu'elle est d'autant plus efficace dans la construction intellectuelle et institutionnelle des cursus d'études proposés et réellement suivis qu'elle y apparait comme banale.

Cette impasse sur la réalité des choses n'est pas que la seule faiblesse de la pensée républicaine. Non seulement il n’y eut pas de remise à plat, quand la III République s'est installée, mais même la notion de disciplines, si déterminante, est entrée dans l'institution scolaire de manière totalement inaperçue. Au moment où il apparut pour désigner l'enseignement, le mot discipline, comme A. Chervel l'a fort bien mis en évidence, fut d'abord utilisé au singulier. Synonyme de gymnastique intellectuelle, il désignait, comme l'écrivait Célestin Hippeau en 1885 cité par Chervel (1988: p. 63), «le développement du jugement, de la raison, de la faculté de combinaison et d'invention ». La discipline n'est pas à elle-même sa propre fin: elle n'est en elle-même qu'un exercice de la raison et de ses facultés. Sa fin est d'instruire le jugement personnel. Le passage au pluriel, sans doute par métonymie, se fit à propos de l'enseignement du secondaire ${ }^{30}$. Nouveau vecteur de cet enseignement, il était certes de nature à en transformer la perspective. Ce qui s'est effectivement produit, mais en partie seulement. Car son introduction pour qualifier des enseignements tels qu'ils existaient ne permit pas, parce qu'implicite, de se rendre compte

28. Non que la question ne soit pas posée, comme le fait par exemple Ernest Renan dans sa célèbre conférence prononcée le 11 mars 1882, Qu'est-ce qu'une nation?, mais personne n'arrive à faire les distinctions nécessaires entre peuple, nation, patrie, État, République....

29. Curriculum construit et curriculum caché ou latent ou implicite, curriculum formel et curriculum réel, curriculum non intentionnel, non enseigné... On trouvera une brève mais claire présentation, ainsi qu'une bibliographie essentielle, dans Forquin (1994) ou De Landsheere (1992, p. 89-119).

30. La plus ancienne attestation que nous ayions trouvée, datant de 1892, fait usage du mot comme synonyme de matières de l'enseignement secondaire, dont la fin est « une forte instruction générale, littéraire, philosophique et scientifique», une "culture intellectuelle et morale» (Basch, 1892). 
que ce que ce terme assumait et prenait en charge n'avait pas grand-chose de républicain : sélection sociale, sélection par le sexe, sélection par le mérite, préparation aux professions libérales, aux emplois publics et à la haute administration, publique ou privée...

\section{La double substitution didactique}

Plus grave encore. Comme la logique qui les portait est celle-là même qui fondait l'épistèmè grecque, la logique de scientificité, le terme en vint très vite à s'étendre également aux sciences elles-mêmes, dont la fin est la production du savoir. De là à penser que la fin des disciplines de formation (initiale, continue ou professionnelle) est le savoir et que ce savoir est un savoir scientifique transposé, c'est faire une double substitution que les didactiques des disciplines n'ont pas hésité à effectuer, jetant tous les acteurs de l'éducation dans le trouble, sinon dans l'opacité la plus complète et, qui plus est, donnant ainsi à croire que la pratique est l'application d'une théorie savante. Or, il nous faut le dire avec insistance, tellement cette conception, répandue comme un slogan indiscutable, entraîne des conséquences fâcheuses dans l'organisation des formations jusqu'au contenu de l'enseignement lui-même (où l'on finit par substituer, dans l'apprentissage de la lecture, l'alphabet phonétique à l'alphabet réel en soutenant que celui-ci est l'alphabet véritable, donc que le monde réel est celui que construit la science!) : il n’y a de théorie qu'à l'intérieur d'une démarche scientifique. Sa validité est interne à cette démarche. Elle est nulle et non avenue en dehors. Il n'y a pas par ailleurs d'usage scientifique des résultats de la science. Aucune action humaine, aucun jugement, aucune décision n'est scientifique. Elle peut être instruite par une investigation qui mette en œuvre une logique de scientificité et qui relève d'une ou de plusieurs sciences. Mais le niveau de discursivité qui fait appel à cette investigation et qui, in fine, exprime un jugement n'est pas scientifique et ne peut pas se couvrir d'une quelconque autorité scientifique. «Personne en son for intérieur ne pense scientifiquement. Tout individu, scientifique ou non, quand il pense un problème, le pense naturellement" aime à répéter Albertini (1992: p. 55). Il n’y a pas de fondement théorique à l'action. Penser le contraire, c'est aboutir, en matière de formation des enseignants, à l'absurdité de vouloir faire de l'enseignant un surhomme, puisqu'il devrait être capable de maîtriser toutes les disciplines savantes (car toutes ont quelque rapport avec l'enseignement) pour pouvoir «fonder théoriquement» son action. La formation didactique des enseignants du primaire qui se décompose en 10 didactiques différentes, parce qu'il y a 10 disciplines enseignées dans le primaire, relève de cette absurdité. 


\section{Par mode de conclusion : de la subversion réciproque à l'émergence d'un vecteur nouveau?}

Dans le monde occidental, l'éducation et la formation des élèves et, par voie de conséquences, la formation des enseignants sont donc globalement envisagées selon deux approches sensiblement différentes : l'une, que spécifie le terme de curriculum, caractérise plutôt le monde anglo-saxon, l'autre, que symbolise le terme de disciplines, le monde français ${ }^{31}$. La première est plutôt sous le régime de la sécularisation de type protestant, la seconde étant plutôt sous celui de la laïcisation, propre au milieu catholique. Curriculum et disciplines, s'ils ne recouvrent pas exactement la même chose, vectorisent, dans chacun de ces deux espaces, la pensée et l'organisation de la formation sous un angle spécifique, sinon opposé : les disciplines, explicitement à partir de l'individu ; le curriculum, explicitement à partir de la société.

\section{Le curriculum comme vecteur intégrateur?}

Que se passe-t-il lorsque deux vecteurs de même ordre se rencontrent? Dans le contexte français, le seul que nous prenons ici comme cadre d'analyse, la réaction qui se produit présentement et qui tend à l'emporter est d'engager totalement la formation dans le vecteur curriculaire, lequel, en révélant tout un monde resté dans l'ombre de l'implicite, s'offre comme un puissant levier de renouvellement et de transformation. Dans ce mouvement, que le langage commun qualifie de mouvement de balancier, il n'y a pas qu'une oscillation d'un extrême à un autre. En fait, ce que le milieu, jusqu'ici porté par la catégorie de disciplines, perçoit dans celle de curriculum, ce n'est pas le curriculum tel qu'il est vécu et pensé dans un pays anglosaxon. Il n'y a pas d'importation directe d'un modèle étranger. Ne connaissant que son vecteur, le vecteur disciplinaire, il part de ce vecteur, mais, au lieu de tirer parti d'une confrontation avec un autre modèle (le modèle curriculaire) pour le soumettre à une critique approfondie, il se laisse aller à construire, sous l'appellation de curriculum, ce qui n'est en fait qu'un contre-modèle de la discipline. Le curriculum qu'il construit repose en fait sur une vision caricaturale du modèle disciplinaire, et non d'une analyse fine de ses caractéristiques. Et c'est cette vision caricaturale qui, inversée, structure le modèle curriculaire ${ }^{32}$.

Dans l'élaboration des référentiels de formation, formes concrètes des curriculums que l'on propose aussi bien pour les élèves que pour leurs futurs enseignants, il y a autant, sinon plus, de la logique du contre-modèle (modèle disciplinaire inversé) que de la logique curriculaire proprement dite. Ce qui revient à dire que les disciplines

31. Cette opposition est constamment l'objet de débats dans les revues et les journaux français. L'analyse très fine qu'en a faite Régis Debray sous le titre "République ou Démocratie » est toujours d'actualité (Debray, 1992, p. 15-54).

32. Nous avons tenté de montrer que l'élaboration conceptuelle de la formation continue se fait selon une même démarche, le vecteur de référence étant le modèle scolaire de la formation (Sachot, 1998c). Mais on pourrait aisément montrer que ce mode de renversement affecte actuellement tous les domaines, y compris la politique. Dans nos attaques contre l'État jacobin et centralisé, ce n'est pas de l'américanisme que nous importons. Nous inversons, en interne, le mouvement jacobin, privant l'État de sa fonction et transférant ce jacobinisme à la région, au local. En aucun cas il ne s'agit d’une importation du modèle américain. 
(scolaires et universitaires) n'y ont plus le statut républicain, mais que, dans la version didactisée que nous venons de critiquer, elles y sont totalement instrumentalisées, mises au service d'objectifs, qui, si détaillés soient-ils, laissent totalement dans l'implicite leurs finalités et les conditions de leur définition, ceux qui les identifient, les procédures utilisées, les passages qui conduisent de leur relevé à la proposition d'une formation et de sa validation. Il n'y a plus de logique républicaine qui traverse l'ensemble du dispositif, le finalise et le hiérarchise, indiquant à chacun dans quel sens diriger son action. Il n'y a plus que du pragmatisme et du réalisme, donnés comme une fidélité à ce qui est. De ce fait, le curriculum, construit à partir d'un référentiel qui atomise ce réel à l'extrême (sans commune mesure avec le morcellement disciplinaire) et se présente comme un simple reflet d'une pratique enseignante évidente, devient en fait l'outil d'un projet de société qui ne dit pas son nom. Parce qu'il rejette dans l'implicite toute sa part intellectuelle (hors la rationalisation), part qui médiatise les rapports entre les individus et la société et qui permet à chaque individu d'exercer son jugement, il peut être considéré comme l'expression aboutie d'une forme sécularisée d'orthodoxie. Il s'impose par lui-même. Le fait devient la vérité et le droit.

\section{La discipline comme vecteur intégrateur?}

Le lecteur aura déjà compris que l'auteur de ces lignes n'est pas près de cautionner une telle démarche, d'accepter que se substitue au vecteur disciplinaire ce qui en est l'inversion caricaturale. Nous estimons que, sauf à faire disparaître le régime républicain, la discipline, comme instruction de la personne pour elle-même, comme logique de scientificité, comme épistémologie grecque (qui est aussi une éthique), reste le vecteur intégrateur de la formation de l'élève comme de celle du professeur qui assure cette formation. C'est la condition sine qua non pour que l'éducation ne sombre pas dans l'inculcation et que la société ne soit l'instance instituante de l'école.

La notion de curriculum, en revanche, peut s'avérer fort précieuse: d'une part, en permettant de lever le voile sur les nombreux implicites qui président de fait à l'éducation et à la formation, initiale ou professionnelle; d'autre part, en invitant à prendre en compte, dans la formation de l'élève, laquelle se prolonge de plus en plus, et d'emblée dans celle de ses maîtres, l'inscription sociale concrète et la préparation à un métier. La confrontation ne devrait pas aboutir, sous peine d'échouer, à la juxtaposition de deux cursus de formation (l'un, qui viserait la formation de l'homme, l'autre celle du futur professionnel) : les savoirs ou savoir-faire préparant à une activité professionnelle (ou plus généralement à la vie sociale) seraient soumis à la logique de scientificité, laquelle est la même, bien qu'elle s'élabore différemment selon les niveaux et les disciplines, au même titre que les savoirs ou savoir-faire identifiés explicitement comme disciplines.

Toute formation est nécessairement curriculaire, d'entrée de jeu. La soumettre au vecteur de la disciplinarité, ainsi que nous l'avons vue se fonder avec la philosophie grecque et s'inscrire dans le système éducatif français avec la III ${ }^{e}$ République, est la seule voie pour faire clairement apparaître - et critiquer - le niveau des finalités 
(reposant sur des principes universels), celui des objectifs (compétences diverses) et celui de la mise en œuvre de la formation (curriculum réel de formation, de validation et de certification), à la rationalisation de laquelle ne peut que s'opposer, à juste titre, la logique disciplinaire. La disciplinarité, qui ne se confond pas avec les nombreuses disciplines universitaires et scientifiques qu'elle génère, retrouve alors sa logique propre et ses exigences contraignantes (comme celle qui impose la maîtrise de procédures spécifiques - les disciplines, justement - et, donc, une inscription durable dans ces procédures au contraire d'un zapping de surface). Sans quoi, la référentialisation (ou opérationnalisation) est à l'éducation et à la formation ce que l'économie de marché est à la société : la mise à l'écart du politique, c'est-à-dire des individus considérés comme sujets dont le jugement a besoin, pour être personnel et autonome, pour résister à l'opinion commune qualifiée abusivement de démocratique, d'être soumis à la logique de scientificité.

\section{Références bibliographiques}

ALBERTINI, J.-M. (1992). La pédagogie n'est plus ce qu'elle sera. Paris : Le Seuil / Presses du CNRS.

BANCEL, D. (1989). Rapport à Lionel Jospin, ministre d'État, ministre de l'Éducation nationale, de la Jeunesse et des Sports, (10 octobre).

BASCH, V. (1892). De l'enseignement des langues modernes. Revue universitaire, t. I, p. 388.

BENVÉNISTE, É. (1966). Catégories de pensée et catégories de langue, dans Problèmes de linguistique générale, I. Paris : Gallimard, coll. «Bibliothèque des sciences humaines», p. 63-74.

BOBBITT, J.F. (1918). The Curriculum. New York: Houghton-Mifflin.

CHERVEL, A. (1988). L'Histoire des disciplines scolaires. Réflexions sur un domaine de recherche. Histoire de l'éducation, ${ }^{\circ}$ 38, p. 59-119.

DEBRAY, R. (1991). Cours de médiologie générale. Paris : Éditions Gallimard, coll. «Bibliothèque des Idées».

DEBRAY, R. (1992). Contretemps. Éloges des idéaux perdus. Paris : Gallimard.

DEBRAY, R. (1997). Transmettre. Paris : Éditions Odile Jacob, coll. «Le champ médiologique».

DEBRAY, R. (2000). Introduction à la médiologie. Paris : PUF, coll. «Premier cycle».

DE LANDSHEERE, V. (1992). L'éducation et la formation. Paris : PUF, coll. «Premier cycle». 
DUMÉZIL, G. (1966). La religion romaine archaïque. Paris : Payot.

ECO, U. (1986). La ligne et le labyrinthe : les structures de la pensée latine, dans D. Duby (dir.), Civilisation latine. Des temps anciens au Monde moderne. Paris : O. Orban, p. 27-57.

FORQUIN, J.-Cl. (1994). Curriculum, dans Ph. Champy et Chr. Étévé (dir.), Dictionnaire encyclopédique de l'éducation et de la formation. Paris : Nathan, $\mathrm{p}$. 218-223.

HABERMAS, J. (1991). La société des individus (traduction de Die Gesellschaft der Individuen. Francfort/Main : Suhrkamp Verlag). Paris : Fayard.

HADOT, P. (1995). Qu'est-ce que la philosophie antique? Paris : Gallimard, coll. «folio/essais".

LELIÈVRE, Cl. (1999). Instruction / Éducation, dans J. Houssaye (coord.), Questions pédagogiques. Encyclopédie historique. Paris : Hachette Éducation, p. 267-275.

LEROI-GOURHAN, A. (1943). Évolution et techniques, t. 1 : L'homme et la matière. Paris : Albin Michel.

LEROI-GOURHAN, A. (1945). Évolution et techniques, t. 2 : Milieu et techniques. Paris : Albin Michel.

LEROI-GOURHAN, A. (1970). Le geste et la parole. La mémoire et les rythmes. Paris : Albin Michel.

LEROI-GOURHAN, A. (1974). Le geste et la parole. Technique et langage. Paris : Albin Michel.

MARBOEUF, Ch. et PRADEAU, J.-F. (1999). Platon. Alcibiade. Paris : GF Flammarion.

MARROU, H.-I. (1965). Histoire de l'éducation dans l'antiquité, 6 e éd. revue et augm. Paris : Éditions du Seuil.

PRADEAU, J.-Fr. (1999). Introduction et notes, dans Ch. Marboeuf et J.-F. Pradeau, Platon. Alcibiade. Paris : GF Flammarion.

RENAN, E. (1982). Qu'est-ce qu'une nation? (Conférence prononcée en 1882), dans H. Psichari (éd.), Euvres complètes, t. I. Paris : Calman-Lévy, 1947, p. 886 et s.

SACHOT, M. (1989). Ouverture, dans M. Sachot (dir.), L'institution de l'histoire, t. II: Mythe, mémoire, fondation. Paris : Cerf/CERIT, p. 13-36.

SACHOT, M. (1991). L'argument d'autorité dans l'enseignement théologique au Moyen Âge : les grandes étapes d'une évolution (XII ${ }^{\mathrm{e}}$-XIII ${ }^{\mathrm{e}}$ siècles), dans $\mathrm{O}$. Reboul et J.-Fr. Garcia (dir.), Rhétorique et pédagogie. Cahiers du séminaire de philosophie, vol. 10. Strasbourg: Presses universitaires de Strasbourg, p. 111-153.

SACHOT, M. (1993). La notion de discipline scolaire : éléments de constitution, dans J.-P. Clément, M. Herr et P. Boyer (dir.), L'identité de l'éducation physique scolaire au XXe siècle. Entre l'école et le sport. Clermont-Ferrand : éd. AFRAPS, p. 127-147. 
SACHOT, M. (1994). Éléments de didactique générale. Strasbourg: Centre de télé-enseignement universitaire.

SACHOT, M. (1996). De la proclamation scripturaire au cours magistral : histoire d'un modèle archétypal, dans Cl. Raisky et $\mathrm{M}$. Caillot (dir.), Au-delà des didactiques, le didactique. Bruxelles : De Boeck-Université, coll. « Perspectives en éducation », p. 193-222.

SACHOT, M. (1997). Disciplines du maître - disciplines de l'élève : contre une "disciplinarisation» du primaire. Cahiers $d u$ CIRID, no 4. (À paraître également dans M. Sachot et Y. Lenoir, Les enseignants du primaire entre disciplines et interdisciplinarité: quelle formation didactique? Bruxelles : De Boeck Université.)

SACHOT, M. (1998a). Discipline générale d'enseignement et référentiel, dans M. Sachot (dir.), Le référentiel d'apprentissage et de formation: un outil didactique?, Strasbourg, CIRID/CRDP d'Alsace, coll. «Recherches didactiques et sciences humaines», vol. 3, p. 67-82.

SACHOT, M. (1998b). L'Invention du Christ. Genèse d'une religion. Paris : Odile Jacob, coll. «Le champ médiologique».

SACHOT, M. (1998c, à paraître). Les disciplines scolaires, modèles ou contremodèles des curricula de formation professionnelle, dans Symposium du REF, Savoirs professionnels et curricula de formation professionnelle. Toulouse, 26-26 octobre 1998.

SACHOT, M. (1999a). La discipline d'enseignement: un singulier pluriel. Essai de déconstruction historique. Documents du Grife, no 1, 1998, p. 1-33; et dans Cahiers du CIRID, n ${ }^{\circ}$ 10, 1999, p. 3-37.

SACHOT, M. (1999b). Religion, dans J. Houssaye (dir.), Questions pédagogiques. Encyclopédie historique. Paris : Hachette éducation, p. 483-509.

SACHOT, M. (2000a). Un discours éclaté. Entre logique disciplinaire et logique curriculaire, «Le discours universitaire». Le Portique, no 6, avril, p. 9-28.

SACHOT, M. (2000b, à paraître). La proclamation scripturaire synagogale, source archétypale du christianisme comme accomplissement du judaïsme.

SMITH, A. (1776). Recherches sur la nature et les causes de la richesse des nations (An Inquiry into the Nature and Causes of the Wealth of Nations), trad.

C. Debyzer. Paris (1947).

TYLER, R. (1950). Basic Principles of Curriculum and Instruction. Chicago : University of Chicago Press.

WITTGENSTEIN, L. (1976). De la certitude. Paris : Gallimard, coll. «TEL». 